\title{
Amino Acids in Conserved Region II Are Crucial to Substrate Specificity, Reaction Velocity, and Regioselectivity in the Transglucosylation of Honeybee GH-13 $\alpha$-Glucosidases
}

\author{
Lukana Ngiwsara, Gaku Iwai, Takayoshi Tagami, Natsuko Sato, Hiroyuki Nakai, ${ }^{*}$ \\ Masayuki Okuyama, Haruhide Mori, ${ }^{\dagger}$ and Atsuo Kimura ${ }^{\dagger}$ \\ Research Faculty of Agriculture, Hokkaido University, Kita-9 Nishi-9, Kita-ku, Sapporo 060-8589, Japan
}

Received June 19, 2012; Accepted July 18, 2012; Online Publication, October 7, 2012

[doi:10.1271/bbb.120473]

Honeybees, Apis mellifera, possess three $\alpha$-glucosidase isozymes, HBG-I, HBG-II, and HBG-III, which belong to glycoside hydrolase family 13. They show high sequence similarity, but clearly different enzymatic properties. HBG-III preferred sucrose to maltose as substrate and formed only $\alpha$-1,4-glucosidic linkages by transglucosylation, while HBG-II preferred maltose and formed the $\alpha-1,6$-linkage. Mutation analysis of five amino acids in conserved region II revealed that Pro226-Tyr227 of HBG-III and the corresponding Asn226-His227 of HBG-II were crucial to the discriminating properties. By replacing these two amino acids, the substrate specificities and regioselectivity in transglucosylation were changed drastically toward the other. The HBG-III mutant, Y227H, and the HBG-II mutant, N226P, which harbor HBG-I-type Pro-His at the crucial positions, resembled HBG-I in enzymatic properties with marked increases in reaction velocities on maltose and transglucosylation ratios. These findings indicate that the two residues are determinants of the enzymatic properties of glycoside hydrolase family 13 $($ GH-13) $\alpha$-glucosidases and related enzymes.

Key words: honeybee $\alpha$-glucosidase; glycoside hydrolase family 13 ; substrate specificity; transglucosylation

$\alpha$-Glucosidase (EC 3.2.1.20, $\alpha$-D-glucoside glucohydrolase) catalyzes the hydrolysis of the non-reducing terminal $\alpha$-glucosidic linkage of substrates to produce $\alpha$-glucose. ${ }^{1,2)}$ It catalyzes transglucosylation as well, depending on the kinds of substrate and their concentrations. It is ubiquitous, and is found in a wide variety of organisms, from microorganisms to higher mammals. The enzymatic properties of $\alpha$-glucosidases are diverse, particularly in substrate specificity. ${ }^{1)}$ Some act preferably on sucrose and aryl $\alpha$-glucosides, and others prefer maltooligosaccharides as substrate. From the structural point of view, most $\alpha$-glucosidases fall into glycoside hydrolase families 13 (GH-13) and 31 according to the CAZy classification of glycoside hydrolases. ${ }^{2,3}$

The European honeybee (Apis mellifera) possesses three $\alpha$-glucosidase isozymes, HBG-I, HBG-II, and
HBG-III. ${ }^{4,5)}$ HBG-I is localized in the ventriculus. HBG-II is present in ventriculus and the hemolymph. HBG-III is secreted from the hypopharyngeal grand into the nectar and is involved in honey formation. ${ }^{6)}$ These enzymes are clearly different in substrate specificity, ability to catalyze transglucosylation, and regioselectivity in transglucosylation. HBG-I shows little or no activity toward nigerose, isomaltose, or soluble starch. ${ }^{7)}$ It catalyzes transglucosylation at higher rates than the others with $\alpha-1,4$ regioselectivity to produce erlose $(\alpha$-Glc- $(1 \rightarrow 4)-\alpha$-Glc- $(1 \leftrightarrow 2)-\beta$-Fru $)$ from sucrose. ${ }^{8)}$ The substrate specificity of HBG-II is so broad that glucobioses having $\alpha-1,2, \alpha-1,3$, and $\alpha-1,6$-glucosidic linkages, phenyl $\alpha$-glucoside, and sucrose are substrates of HBG-II besides maltooligosaccharides. ${ }^{9)}$ HBG-I and HBG-II exhibit non-Michaelis-Menten kinetics, which has been considered allosteric behavior of the enzymes even though they are monomeric enzymes. ${ }^{7,9)}$ For instance, HBG-I cleaves the linkage of maltose, sucrose, and 4-nitrophenyl $\alpha$-glucoside in a mode of negative cooperativity, ${ }^{7)}$ whereas HBG-II shows positive cooperativity. ${ }^{9)}$ HBG-III, on the other hand, follows typical Michaelis-Menten kinetics. It preferentially hydrolyzes sucrose. The values of $k_{\text {cat }}$ and $K_{\mathrm{m}}$ of the enzyme on several substrates are higher than those of HBG-I and HBG-II. ${ }^{5)} \alpha$-Glucosidase isozymes are also found in a Japanese subspecies of eastern honeybee, Apis cerena japnonica. One of them, JBG-I, displays non-MichaelisMenten type kinetic behavior, as observed for HBG-I. ${ }^{10)}$

In spite of the differences in enzymatic properties, honeybee $\alpha$-glucosidases belong to GH-13 and exhibit considerable sequence similarity to each other. ${ }^{11,12)}$ HBG-III shares $57 \%$ and $64 \%$ identical amino acids with HBG-I and HBG-II respectively.

GH-13 includes a wide variety of $\alpha$-glucoside-active enzymes, which catalyze hydrolysis and transglucosylation with retention of the anomeric configuration of the substrates. ${ }^{3,13)}$ For instance, $\alpha$-amylase (EC 3.2.1.1), pullulanase (EC 3.2.1.41), cyclodextrin glucanotransferase (EC 2.4.1.19), and neopullulanase (EC 3.2.1.135) are GH-13 enzymes. Exo-glucoside-acting enzymes, such as oligo-1,6-glucosidase (EC 3.2.1.10), dextran glucosidase (EC 3.2.1.70), amylosucrase (EC 2.4.2.4),

\footnotetext{
To whom correspondence should be addressed. Haruhide MoRI, Tel: +81-11-706-2816; Fax: +81-11-706-2808; E-mail: haru@abs.agr.

hokudai.ac.jp; Atsuo KIMURA, Tel/Fax: +81-11-706-2808; E-mail: kimura@abs.agr.hokudai.ac.jp

* Present address: Faculty of Agriculture, Niigata University, Niigata 950-2181, Japan

Abbreviations: GH-13, glycoside hydrolase family 13; HPAEC-PAD, high performance anion-exchange chromatography-pulsed amperometric detection
} 
sucrose phosphorylase (EC 2.4.1.7), and isomaltulose synthase (EC 5.4.99.11), are also included in the family. GH-13 enzymes share three domains, A, B, and C, in protein structure. The active sites are in a cleft or a pocket formed by $(\beta / \alpha)_{8}$ barrel-shaped catalytic domain $\mathrm{A}$ and protruding domain $\mathrm{B}$ inserted between the third $\beta$-stand and the third $\alpha$-helix of domain A. The four conserved sequence regions, I-IV, ${ }^{13)}$ which are located in $\beta$-strands $3,4,5$, and 7 of the $(\beta / \alpha)_{8}$ barrel or their C-terminal extensions, are deeply involved in catalysis and substrate binding in that they form subsite -1 . In particular, region II and region III include Asp (Asp223 of both HBG-II and HBG-III) and Glu (Glu292 of HBGII and Glu286 of HBG-III), ${ }^{12)}$ which are considered to play the role of nucleophile and that of general acid/ base in the catalytic reaction, respectively. The residues following the two catalytic amino acids are thought to play important roles, in substrate binding at the +1 and +2 subsites in GH-13 enzymes. ${ }^{14-18)}$

In this study, to identify the amino acids responsible for the specific enzymatic properties of HBGs, we focused on the amino acid residues in region II (Fig. 1). Using HBG-II and HBG-III, which have been successfully produced heterologously in Pichia pastoris, ${ }^{12)}$ the residues were replaced by the corresponding amino acids of the other isozymes. The site-directed mutation, particularly at positions equivalent to Pro226-Tyr227 of HBG-III, caused drastic changes in substrate specificity. Their drastic change in regioselectivity in transglucosylation is also described below, together with the nature of the wild-type HBG-II and HBG-III.

\section{Materials and Methods}

Substrates. Maltose and sucrose were purchased from Nacalai Tesque (Kyoto, Japan). Kojibiose, nigerose, maltotriose, and isomaltulose were from Wako Pure Chemical Industries (Osaka, Japan). Isomaltose and panose $(\alpha$-Glc- $(1 \rightarrow 6)-\alpha$-Glc- $(1 \rightarrow 4)$-Glc) were from Tokyo Chemical Industry (Tokyo). Turanose $(\alpha$-Glc- $(1 \rightarrow 3)$-Fru) was from ICN Biomedicals (Irvine, CA), maltulose was from Alfa Aesar (Ward Hill, MA), and erlose was from Hayashibara Biochemical Laboratories (Okayama, Japan).

$\begin{array}{ll}\text { HBG-III } & \text { 223-DALPYIC } \\ \text { HBG-II } & \text { 223-DAINHMF } \\ \text { HBG-I } & \text { 230-DAVPHLF } \\ \text { Taka-amylase } & \text { 206-DTVKHVQ } \\ \text { B. sub } \alpha \text {-amylase } & \text { 176-DAAKHIE } \\ \text { Sucrose hydrolase (SUH) } & \text { 280-DSTAYLW } \\ \text { Sucrose phosphorylase (SucP) } & \text { 192-DAVGYGA } \\ \text { Amylosucrase } & \text { 286-DAVAFIW } \\ \text { Trehaluose synthase (MutB) } & \text { 200-DTVATYS } \\ \text { SmDG } & \text { 194-DVIDMIG }\end{array}$

Fig. 1. Partial Amino Acid Sequences of Conserved Region II.

The catalytic Asp, indicated by an asterisk, and the adjacent amino acids are shown. Amino acids mutated in this study are underlined. Pro226-Tyr227 of HBG-III and their equivalents are indicated by bold-faced letters. The partial sequences, listed from the top, are of $\alpha$-glucosidase isozymes III, II, and I from Apis mellifera (HBG-III, HBG-II, and HBG-I respectively), ${ }^{6,12)}$ Taka-amylase from Aspergillus oryzae, ${ }^{25)}$ Bacillus subtilis $\alpha$ amylase, ${ }^{26)}$ sucrose hydrolase SUH from Xanthomonas axonopodis ${ }^{27)}$ sucrose phosphorylase from Bifidobacterium adolescentis, ${ }^{28)}$ amylosucrase from Neisseria polysaccharea, ${ }^{30)}$ trehalulose synthase (MutB) from Psuedomonas mesoacidophila, ${ }^{31)}$ and dextran glucosidase (SmDG) from Streptococcus mutans. ${ }^{29)}$
Strains and plasmid. Escherichia coli DH5 $\alpha$ (Stratagene, La Jolla, CA) was used for plasmid preparation. Pichia pastoris GS115 (his4, $\mathrm{Mut}^{+}$) (Invitrogen, Carlsbad, CA) was used as host for the enzyme production. For HBG-III production, a pPICZ $\alpha \mathrm{A}$ derivative (Invitrogen) harboring the cDNA coding for mature HBG-III (Ala18-Phe567) between the EcoRI and NotI sites was used. The ORF encoded mature HBG-III equipped with an 89-amino acid long $\alpha$-factor signal sequence, which was linked to its $\mathrm{N}$-terminus by two amino acids, E-F, at the joint. The HBG-II production plasmid was a pPICZA derivative (Invitrogen) harboring the HBG-II cDNA between the EcoRI and NotI sites of the plasmid. The start codon and the stop codon of the HBG-II cDNA were placed adjacent to the EcoRI site and the NotI site of pPICZA respectively.

Mutagenesis. Site-directed mutagenesis was done using a PrimeSTAR Mutagenesis Kit (Takara Bio, Otsu, Japan). Specific oligonucleotides were designed following the manufacturer's instructions to generate mutations, as follows: In HBG-III, Leu225 (CTG) $\rightarrow$ Ile (ATC)/Val (GTA), Pro226 (CCT) $\rightarrow$ Asn (AAC), Tyr227 (TAC) $\rightarrow$ His (CAC), Ile228 (ATT) $\rightarrow$ Met (ATG)/Leu (TTA), and Cys229 $(\mathrm{TGC}) \rightarrow$ Phe (TTC). In HBG-II, Asn226 (AAC) $\rightarrow$ Pro (CCA), and His227 (CAC) $\rightarrow$ Tyr (TAC). The PCR mixture was used directly in the transformation of $E$. coli DH5 $\alpha$. Plasmids were prepared, and the sequences of the ORFs and the connections were verified using a BigDye Terminator v3.1 Cycle Sequencing Kit and an ABI PRISM 310 DNA sequencer (Applied Biosystems, Foster City, CA).

Transformation of $\mathrm{P}$. pastoris. The expression plasmids were linearized by restriction digestion with $\mathrm{SacI}$ and used in the transformation of $P$. pastoris GS115. Competent cells were prepared by the method of Lin-Cereghino et al., ${ }^{19)}$ with some modifications. Briefly, $P$. pastoris was grown in $10 \mathrm{~mL}$ of YPD (1\% yeast extract, $2 \%$ peptone, and $2 \%$ glucose) until $A_{600}$ reached 1 . First the cells were incubated for $5 \mathrm{~min}$ at $30^{\circ} \mathrm{C}$ in $1.8 \mathrm{~mL}$ of BEDS solution $(10 \mathrm{~mm}$ Bicine- $\mathrm{NaOH} \mathrm{pH} 8.3,3 \% \mathrm{v} / \mathrm{v}$ ethylene glycol, $5 \% \mathrm{v} / \mathrm{v}$ dimethyl sulfoxide, and $1 \mathrm{M}$ sorbitol), supplemented with $0.2 \mathrm{~mL}$ of $1 \mathrm{M}$ dithiothreitol (DTT), and then resuspended in $50 \mu \mathrm{L}$ of BEDS solution without DTT. The linearized plasmid DNA was mixed with the competent cells $(55 \mu \mathrm{L})$ in a $1-\mathrm{mm}$ gapped cuvette, and an electroporation pulse was applied at $1.5 \mathrm{kV}, 200 \Omega$, and $25 \mu \mathrm{F}$ (GenePulser II, Bio-Rad, Hercules, CA). The transformants were screened on YPDS agar plates (1\% yeast extract, $2 \%$ peptone, $2 \%$ glucose, $1 \mathrm{M}$ sorbitol, and $2 \%$ agar) containing $100 \mu \mathrm{g} / \mathrm{mL}$ of zeocin. Single colonies were streaked onto an $\mathrm{MDH}$ agar plate $(1.34 \%$ yeast nitrogen base with ammonium sulfate and without amino acids (Difco, Franklin Lakes, $\mathrm{NJ}), 4 \mu \mathrm{g} / \mathrm{mL}$ of D-biotin, $2 \%$ glucose, and $1.5 \%$ agar) and stored at $4{ }^{\circ} \mathrm{C}$.

Enzyme preparation. P. pastoris transformants were grown in BMGY medium (as MDH, but $1 \%$ glycerol replaced $2 \%$ glucose, and with the addition of $1 \%$ yeast extract, $2 \%$ peptone, and $0.1 \mathrm{M}$ potassium phosphate buffer, $\mathrm{pH}$ 6.0) with shaking at $30^{\circ} \mathrm{C}$ for $24 \mathrm{~h}$. Then the cells were resuspended in BMMY (as BMGY, but $0.5 \%$ methanol replaced $1 \%$ glycerol) and shaken continuously for $72 \mathrm{~h}$ at $30^{\circ} \mathrm{C}$. The recombinant enzymes were purified from the culture supernatant by ammonium sulfate precipitation at $90 \%$ saturation and anion-exchange chromatography on a DEAE Sepharose Fast Flow column $(3 \times 15 \mathrm{~cm}$, $100 \mathrm{~mL}$ ) and a 6-mL Resource-Q column (GE Healthcare, Little Chalfont, UK) with a BioCAD 700E HPLC system (Applied Biosystems). Both columns were pre-equilibrated with $20 \mathrm{~mm}$ sodium phosphate buffer ( $\mathrm{pH}$ 7.8). The protein samples were dialyzed against the same buffer in advance and loaded onto the columns. The adsorbed proteins were eluted with a linear gradient of 0 to $0.12 \mathrm{M} \mathrm{NaCl}$ in the first chromatography, and with combined gradients of $\mathrm{NaCl}(0$ to $0.12 \mathrm{M}$ ) and $\mathrm{pH}(\mathrm{pH} 7.8$ to 6.0 ) in the second. In both chromatographies, active fractions were pooled. Using Centriprep YM-30 (Millipore, Billerica, MA), the protein was concentrated and the buffer was replaced with $20 \mathrm{~mm}$ sodium phosphate buffer ( $\mathrm{pH} 7.8$ ). The homogeneity of the purified enzymes was confirmed by SDS$\mathrm{PAGE}^{20)}$ on $8 \%$ polyacrylamide gel. Proteins were stained by Rapid CBB Kanto (Kanto Chemical, Tokyo).

Enzyme and protein assays. The protein concentrations were determined by quantification of the various amino acids of protein 
hydrolysate $\left(6 \mathrm{~N} \mathrm{HCl}\right.$ at $110^{\circ} \mathrm{C}$ for $\left.24 \mathrm{~h}\right)$ by the ninhydrin colorimetric method using an amino acid analyzer (AminoTac JLC/500V, JEOL, Tokyo).

Enzyme activity (1 U) was defined as the amount of enzyme that catalyzes the formation of glucose at an initial rate of $2 \mu \mathrm{mol} \mathrm{min}{ }^{-1}$ from $5.8 \mathrm{~mm}$ maltose under standard reaction conditions. The reaction mixture $(50 \mu \mathrm{L})$, composed of $5.8 \mathrm{~mm}$ maltose, $44 \mathrm{~mm}$ sodium acetate buffer ( $\mathrm{pH} 5.5$ ), $0.022 \%$ Triton $\mathrm{X}-100$, and an adequate concentration of the enzyme, was incubated at $37^{\circ} \mathrm{C}$ for $10 \mathrm{~min}$. The enzymatic reaction was terminated by the addition of $100 \mu \mathrm{L}$ of $2 \mathrm{M}$ Tris $-\mathrm{HCl}$ (pH 7.0), which inhibited activity completely, and glucose concentrations were measured by the glucose oxidase-peroxidase method ${ }^{21)}$ using Glucose C-II Test Wako (Wako Pure Chemical Industries).

The substrate specificities of the enzymes were analyzed by measuring glucose-releasing velocities from various saccharides under standard reaction conditions. The substrate concentrations were $1 \mathrm{~mm}$ for HBG-II, and $5 \mathrm{~mm}$ for HBG-III. Isomaltose was $20 \mathrm{~mm}$ only for HBG-III.

Kinetic constant determination. The initial velocities of glucose liberation from sucrose and maltose were determined over a wide range of substrate concentrations up to $150 \mathrm{~mm}$. The reactions were done under standard assay conditions. The ranges of substrate concentrations, in which a linear relationship was observed in Lineweaver-Burk plots, were determined first, and then kinetic parameters $K_{\mathrm{m}}$ and $k_{\text {cat }}$ were determined by non-linear regression to the Michaelis-Menten equation using KaleidaGraph software (Synergy Software, Reading, PA). In each experiment, the enzymes were diluted to an appropriate concentration to determine initial reaction velocities. The sucrose concentrations used for the HBG-III derivatives were 10 $150 \mathrm{~mm}$ (L225I, L225V, and L225I-P226N), 2-100 mm (the wild type, P226N, P226N-H227Y, I228M, and C229F), 4-80 mM (I228L), 2$60 \mathrm{~mm}$ (Y227H-I228L), 2-40 mm (Y227H), and 2-30 mm (Y227HI228M). Sucrose for the HBG-II derivatives was 5-60 mM (H227Y), 5-20 mm (N226P), and 5-30 mM (wild-type HBG-II, N226P-H227Y). Maltose for HBG-III was ranging in 4-150 mM (L225I, L225V, I228L), 4-100 mM (L225I-P226N, P226N, I228M), 4-50 mM (wildtype HBG-III, C229F), and 0.2-7 mM (Y227H, P226N-Y227H, Y227H-I228L/M). For HBG-II, maltose of 6-150 mM (N226P$\mathrm{H} 227 \mathrm{Y}), \quad 6-30 \mathrm{~mm}$ (H227Y), $0.5-5 \mathrm{~mm}$ (N226P), and $0.5-3 \mathrm{~mm}$ (wild-type HBG-II) was used. Maltotriose for HBG-III was 0.2$15 \mathrm{~mm}$ (Y227H, Y227H-I228M), 0.5-20 mM (Y227H-I228L), 2-50 mM (C229F), 2-80 mM (wild type, L225I/V, P226N, I228M), 5-80 mM (I228L, L225I-P226N), and 2-150 mM (P226N-Y227H). For HBG-II, maltotriose was $0.5-1.5 \mathrm{~mm}(\mathrm{~N} 226 \mathrm{P}), 0.5-3 \mathrm{~mm}$ (wild type), $1-10 \mathrm{~mm}$ (N226P-H227Y), and 0.5-150 mM (H227Y). All experiments were performed in triplicate, and averages and standard deviations were calculated.

The transglucosylation ratio, $r_{\mathrm{tg}}$, was defined as previously: ${ }^{22)}$

$$
r_{\mathrm{tg}}=v_{\mathrm{tg}} /\left(v_{\mathrm{tg}}+v_{\mathrm{h}}\right)
$$

where $v_{\mathrm{tg}}$ and $v_{\mathrm{h}}$ are initial reaction velocities of transglucosylation and hydrolysis respectively.

Analysis of transglucosylation by TLC. Maltose and sucrose $(150 \mathrm{~mm})$ were incubated with $22 \mu \mathrm{g} \mathrm{mL}^{-1}$ of enzyme under standard reaction conditions for $15 \mathrm{~min}$ to $6 \mathrm{~h}$, and the reaction mixtures were heated at $95^{\circ} \mathrm{C}$ for $3 \mathrm{~min}$ to terminate the reaction. The solution was diluted 5-fold, and $2 \mu \mathrm{L}$ was spotted onto a Silica gel 60 plate (Merck, Darmstadt, Germany). Carbohydrates were developed by a solvent system of nitromethane:1-propanol:water $(2: 5: 1.5, v / v)$, and were visualized with a methanol solution containing $0.03 \%$ 1-naphthol and $5 \%(w / v)$ sulfuric acid and heating.

Analysis of transglucosylation by HPAEC-PAD. The reaction products of $150 \mathrm{~mm}$ maltose and sucrose were analyzed by high performance anion-exchange chromatography-pulsed amperometric detection (HPAEC-PAD). The enzyme reaction was done under standard conditions, and was stopped by heating at $100^{\circ} \mathrm{C}$ for $3 \mathrm{~min}$. The reaction time was $3 \mathrm{~min}$ to $5 \mathrm{~h}$. Quantification was done by HPAEC-PAD using a Dionex ICS-3000 system equipped with a CarboPac PA-1 column $(\phi 4 \times 250 \mathrm{~mm}$; Dionex, Sunnyvale, CA $)$. Elution was performed at a flow rate of $0.8 \mathrm{~mL} \mathrm{~min}^{-1}$ at $25^{\circ} \mathrm{C}$ using
$640 \mathrm{~mm}$ sodium hydroxide as isocratic mobile phase. The reaction mixture was adequately diluted with water, and $10 \mu \mathrm{L}$ was injected. The reaction products were identified based on retention times and were quantified from areas of peaks in the comparison with standard carbohydrates $(5-200 \mu \mathrm{M})$.

\section{Results}

Enzyme production and specific activity

Honeybee $\alpha$-glucosidase isozymes II and III (HBG-II and HBG-III) were heterologously produced in Pichia pastoris. The ORFs encoded respectively the full-length immature HBG-II containing the original N-terminal signal sequence for secretion of the protein and mature HBG-III (Ala18-Phe567 of immature HBG-III) with the $\alpha$-factor signal sequence at the $\mathrm{N}$-terminus and two additional amino acids, Glu-Phe, in the joint part. After $72 \mathrm{~h}$ of induction, the enzymes were accumulated in $1 \mathrm{~L}$ of the supernatant to $184 \mathrm{U}$ for HBG-II and $83 \mathrm{U}$ for HBG-III, which were calculated to be $12 \mathrm{mg} / \mathrm{L}$ and $7.0 \mathrm{mg} / \mathrm{L}$ respectively by the specific activities of the purified enzymes (Table 1).

Five amino acids in conserved region II, Leu225, Pro226, Tyr227, Ile228, and Cys229, of HBG-III were mutated. Seven single mutant HBG-III enzymes, L225I/ $\mathrm{V}, \mathrm{P} 226 \mathrm{~N}, \mathrm{Y} 227 \mathrm{H}, \mathrm{I} 228 \mathrm{~L} / \mathrm{M}$, and $\mathrm{C} 229 \mathrm{~F}$, and four double mutants, L225I-P226N, P226N-Y227H, and Y227H-I228L/M, were generated. Counter-mutants of HBG-II, N226P, H227Y, and N226P-H227Y, were prepared. All the mutant enzymes were produced as wild-type enzymes with similar yields $(4.5 \mathrm{mg} / \mathrm{L}$ for Y227H-I228L to $54 \mathrm{mg} / \mathrm{L}$ for $\mathrm{P} 226 \mathrm{~N}$ in the HBG-III mutants, and $3.9 \mathrm{mg} / \mathrm{L}$ for $\mathrm{H} 227 \mathrm{Y}$ to $12 \mathrm{mg} / \mathrm{L}$ for N226P-H227Y in the HBG-II mutants). In the SDSPAGE analyses, all of the enzymes, purified from the supernatant, showed a single protein band migrating to the positions of $68 \mathrm{kDa}$ and $76 \mathrm{kDa}$ respectively, as native HBG-III and HBG-II. The specific activities of recombinant wild-type HBG-III and HBG-II for $5.8 \mathrm{~mm}$ maltose were $5.90 \mathrm{U} / \mathrm{mg}$ and $7.75 \mathrm{U} / \mathrm{mg}$ respectively (Table 1).

The introduction of mutations in HBG-III markedly affected the specific activity. Single mutant HBG-III, $\mathrm{P} 226 \mathrm{~N}$, and two double mutants containing the $\mathrm{P} 226 \rightarrow \mathrm{N}$ mutation showed lower activity than the wild type. In contrast, $\mathrm{Y} 227 \mathrm{H}$ and the combination of it with $\mathrm{I} 228 \mathrm{~L} / \mathrm{M}$ exhibited drastic increases in activity (Table 1). Two single mutants of HBG-II, N226P and $\mathrm{H} 227 \mathrm{Y}$, showed very high activities, but double mutant N226P-H227Y decreased in specific activity to a level similar to wild-type HBG-III (Table 1).

\section{Substrate specificity}

The substrate preferences of the enzymes for disaccharides were investigated (Fig. 2). Wild-type HBG-III showed high preference for sucrose with the highest initial reaction velocity among the substrates at $5 \mathrm{~mm}$. Maltose was consumed with $24 \%$ of the velocity of sucrose. Kojibiose, nigerose, isomaltose, turanose, and maltulose were poor substrates. Wild-type HBG-II, on the other hand, exhibited broad substrate specificity at a fixed concentration of $1 \mathrm{~mm}$ (Fig. 2). The best substrate for HBG-II was maltose, with 2.3-fold higher initial velocity than sucrose. Kojibiose, isomaltose, turanose, and isomaltulose were catalyzed as fast as 
Table 1. Kinetic Parameters of Hydrolysis at Low Concentrations of Substrates

\begin{tabular}{|c|c|c|c|c|c|c|c|c|c|c|}
\hline \multirow[b]{2}{*}{ Enzyme } & \multirow{2}{*}{$\begin{array}{l}\text { Specific } \\
\text { activity }^{\mathrm{a}} \\
(\mathrm{U} / \mathrm{mg})\end{array}$} & \multicolumn{3}{|c|}{ Sucrose } & \multicolumn{3}{|c|}{ Maltose } & \multicolumn{3}{|c|}{ Maltotriose } \\
\hline & & $\begin{array}{l}k_{\text {cat }} \\
\left(\mathrm{s}^{-1}\right)\end{array}$ & $\begin{array}{c}K_{\mathrm{m}} \\
(\mathrm{mM})\end{array}$ & $\begin{array}{c}k_{\text {cat }} / K_{\mathrm{m}} \\
\left(\mathrm{s}^{-1} \mathrm{~mm}^{-1}\right)\end{array}$ & $\begin{array}{l}k_{\text {cat }} \\
\left(\mathrm{s}^{-1}\right)\end{array}$ & $\begin{array}{c}K_{\mathrm{m}} \\
(\mathrm{mM})\end{array}$ & $\begin{array}{c}k_{\mathrm{cat}} / K_{\mathrm{m}} \\
\left(\mathrm{s}^{-1} \mathrm{~mm}^{-1}\right)\end{array}$ & $\begin{array}{l}k_{\text {cat }} \\
\left(\mathrm{s}^{-1}\right)\end{array}$ & $\begin{array}{c}K_{\mathrm{m}} \\
(\mathrm{mM})\end{array}$ & $\begin{array}{c}k_{\text {cat }} / K_{\mathrm{m}} \\
\left(\mathrm{s}^{-1} \mathrm{~mm}^{-1}\right)\end{array}$ \\
\hline \multicolumn{11}{|l|}{ HBG-III } \\
\hline Wild type & 5.90 & $222 \pm 10$ & $42.3 \pm 2.8$ & $5.27 \pm 0.12$ & $47.2 \pm 1.5$ & $14.0 \pm 1.7$ & $3.40 \pm 0.31$ & $133 \pm 1$ & $8.56 \pm 0.16$ & $15.6 \pm 0.4$ \\
\hline $\mathrm{L} 225 \mathrm{~V}$ & 7.45 & $332 \pm 3$ & $73.6 \pm 2.1$ & $4.51 \pm 0.1$ & $101 \pm 2$ & $34.7 \pm 1.7$ & $2.91 \pm 0.08$ & $257 \pm 6$ & $19.2 \pm 0.7$ & $13.4 \pm 0.3$ \\
\hline L225I & 6.25 & $159 \pm 3$ & $49.6 \pm 2.2$ & $3.20 \pm 0.09$ & $50.8 \pm 0.8$ & $22.8 \pm 0.9$ & $2.23 \pm 0.05$ & $177 \pm 6$ & $14.6 \pm 1.1$ & $12.2 \pm 0.6$ \\
\hline $\mathrm{P} 226 \mathrm{~N}$ & 1.50 & $24.8 \pm 1.1$ & $13.9 \pm 3.1$ & $1.86 \pm 0.34$ & $6.19 \pm 0.4$ & $30.8 \pm 0.9$ & $0.201 \pm 0.012$ & $18.9 \pm 1.4$ & $6.43 \pm 0.58$ & $2.98 \pm 0.47$ \\
\hline $\mathrm{Y} 227 \mathrm{H}$ & 56.5 & $100 \pm 5$ & $17.5 \pm 1.9$ & $5.78 \pm 0.49$ & $118 \pm 2$ & $2.43 \pm 0.03$ & $48.3 \pm 0.4$ & $117 \pm 4$ & $1.63 \pm 0.14$ & $72.2 \pm 5.8$ \\
\hline $\mathrm{I} 228 \mathrm{~L}$ & 4.55 & $231 \pm 12$ & $37.2 \pm 1.7$ & $6.19 \pm 0.05$ & $37.6 \pm 0.5$ & $23.0 \pm 0.2$ & $1.65 \pm 0.04$ & $127 \pm 0$ & $9.69 \pm 0.09$ & $13.1 \pm 0.1$ \\
\hline $\mathrm{I} 228 \mathrm{M}$ & 3.73 & $181 \pm 2$ & $34.6 \pm 0.5$ & $5.24 \pm 0.12$ & $26.1 \pm 0.9$ & $25.0 \pm 0.6$ & $1.04 \pm 0.05$ & $97.4 \pm 0.4$ & $10.0 \pm 0.7$ & $9.77 \pm 0.65$ \\
\hline $\mathrm{C} 229 \mathrm{~F}$ & 12.0 & $145 \pm 14$ & $17.0 \pm 1.5$ & $8.61 \pm 1.05$ & $69.0 \pm 6.3$ & $14.8 \pm 0.5$ & $4.68 \pm 0.44$ & $107 \pm 4$ & $8.12 \pm 0.2$ & $13.2 \pm 0.8$ \\
\hline L225I-P226N & 1.30 & $89.8 \pm 2.2$ & $20.3 \pm 1.5$ & $4.45 \pm 0.43$ & $24.0 \pm 1.4$ & $74.2 \pm 2.7$ & $0.324 \pm 0.019$ & $64.4 \pm 2.0$ & $10.8 \pm 0.6$ & $5.97 \pm 0.25$ \\
\hline P226N-Y227H & 0.800 & $0.522 \pm 0.076$ & $15.5 \pm 1.5$ & $0.0341 \pm 0.0059$ & $0.325 \pm 0.027$ & $0.780 \pm 0.240$ & $0.452 \pm 0.118$ & $0.453 \pm 0.021$ & $0.646 \pm 0.093$ & $0.711 \pm 0.071$ \\
\hline Y227H-I228L & 66.5 & $95.3 \pm 5.8$ & $17.0 \pm 1.9$ & $5.67 \pm 0.66$ & $166 \pm 3$ & $4.13 \pm 0.29$ & $40.4 \pm 2.1$ & $183 \pm 4$ & $2.32 \pm 0.02$ & $78.9 \pm 2.1$ \\
\hline Y227H-I228M & 34.8 & $73.3 \pm 6.1$ & $12.2 \pm 3.0$ & $6.55 \pm 2.20$ & $106 \pm 8$ & $2.78 \pm 0.26$ & $38.4 \pm 1.6$ & $111 \pm 3$ & $1.47 \pm 0.07$ & $75.4 \pm 5.7$ \\
\hline \multicolumn{11}{|l|}{ HBG-II } \\
\hline Wild type & 7.75 & $87.6 \pm 12.5$ & $30.6 \pm 5.7$ & $2.91 \pm 0.34$ & $37.7 \pm 2.1$ & $4.02 \pm 0.37$ & $9.43 \pm 0.41$ & $87.2 \pm 3.3$ & $3.82 \pm 0.14$ & $22.8 \pm 0.1$ \\
\hline $\mathrm{N} 226 \mathrm{P}$ & 20.6 & $199 \pm 3$ & $13.4 \pm 0.4$ & $14.8 \pm 0.5$ & $108 \pm 9$ & $2.74 \pm 0.19$ & $39.4 \pm 2.1$ & $137 \pm 8$ & $2.16 \pm 0.15$ & $63.2 \pm 1.6$ \\
\hline $\mathrm{H} 227 \mathrm{Y}$ & 15.4 & $211 \pm 6$ & $45.6 \pm 2.2$ & $4.65 \pm 0.36$ & $146 \pm 11$ & $26.4 \pm 2.9$ & $5.57 \pm 0.18$ & $244 \pm 3$ & $16.9 \pm 1.1$ & $14.5 \pm 1.1$ \\
\hline N226P-N227Y & 5.85 & $231 \pm 11$ & $24.9 \pm 2.7$ & $9.34 \pm 0.63$ & $97.4 \pm 2.3$ & $24.8 \pm 0.2$ & $3.93 \pm 0.13$ & $203 \pm 13$ & $14.5 \pm 0.9$ & $14.0 \pm 0.3$ \\
\hline
\end{tabular}

Parameters were determined in a range of substrate concentrations that gave a linear relationship between $1 / s$ and $1 / v$.

${ }^{a}$ Half of glucose-releasing velocity on $5.8 \mathrm{~mm}$ maltose.

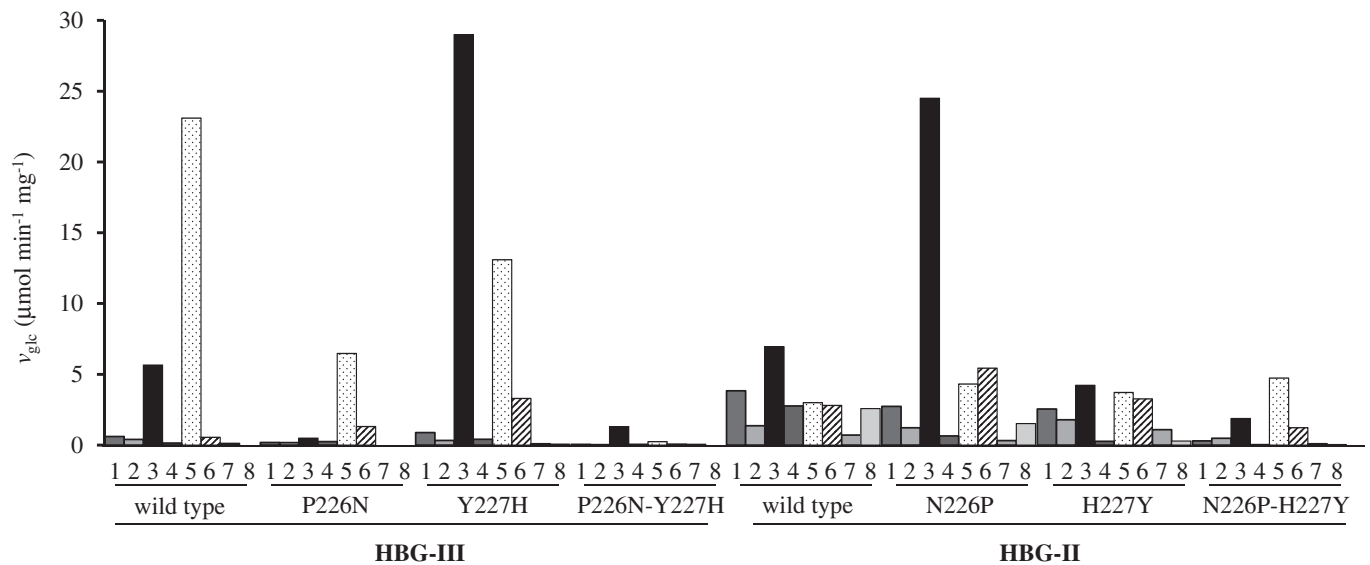

Fig. 2. Substrate Preference of Wild-Type and Mutant HBG-III and HBG-II.

Initial glucose-releasing velocities for various disaccharides are shown. 1, kojibiose; 2, nigerose; 3, maltose; 4, isomaltose; 5, sucrose; 6, turanose; 7 , maltulose; 8 , isomaltulose. The substrate concentration was $5 \mathrm{~mm}$ for HBG-III and $1 \mathrm{~mm}$ for HBG-II.

sucrose. Nigerose and maltulose were also catalyzed, with $20 \%$ and $10 \%$ of the initial rate of maltose respectively.

The sucrose preference of wild-type HBG-III was considerably changed by the mutations (Fig. 2). The $\mathrm{P} 226 \mathrm{~N}$ mutant showed significantly reduced reaction rates for sucrose and maltose, while its rates for turanose and isomaltose increased 2.4- and 1.8-fold respectively, and hence turanose was a better substrate than maltose for the $\mathrm{P} 226 \mathrm{~N}$ mutant. The $\mathrm{Y} 227 \rightarrow \mathrm{H}$ mutation reduced the activity for sucrose to $57 \%$, but caused a drastic increase in the reaction rates for maltose, isomaltose, and turanose, by 2.9- to 6.0-fold. At a concentration of $5 \mathrm{mM}$, maltose was the best substrate for Y227H. Double mutant $\mathrm{P} 226 \mathrm{~N}-\mathrm{Y} 227 \mathrm{H}$ showed marked reduction of all rates, but preferred maltose as substrate at a 5-times higher reaction velocity than sucrose. The other disaccharides tested were poor substrates for the three HBG-III mutants as observed for the wild type.

The mutations of HBG-II also affected substrate specificity (Fig. 2). N226P showed high preference for maltose, with 3.5-fold elevated velocity for it, together with decreased velocities for isomaltose, and isomaltulose by 4.3 - and 1.7-fold respectively. In the mutants related to $\mathrm{H} 227 \mathrm{Y}$, the velocities on maltose were lower, and the sucrose-acting rates were increased. In particular, N226P-H227Y catalyzed sucrose with $158 \%$ of the velocity of the wild type, while all of the other rates decreased to $44 \%$ or less. For the double mutant, sucrose was the most preferred substrate.

\section{Kinetic parameters}

The kinetic parameters for sucrose, maltose, and maltotriose were determined at low ranges of substrate concentrations, giving an apparently linear relationship between $1 / v$ and $1 /[\mathrm{s}]$ (Table 1). Maltotriose was the best substrate for both wild-type HBG-III and HBG-II among the three substrates with respect to the highest $k_{\text {cat }} / K_{\mathrm{m}}$. Wild-type HBG-III preferred sucrose to maltose. Sucrose was catalyzed with the highest $k_{\text {cat }}$, but the $K_{\mathrm{m}}$ for it was also very high. On the other hand, wildtype HBG-II preferred maltose to sucrose with respect to $k_{\text {cat }} / K_{\mathrm{m}}$, due mainly to a $K_{\mathrm{m}}$ as low as that for maltotriose. Wild-type HBG-II showed lower $k_{\text {cat }}$ and 

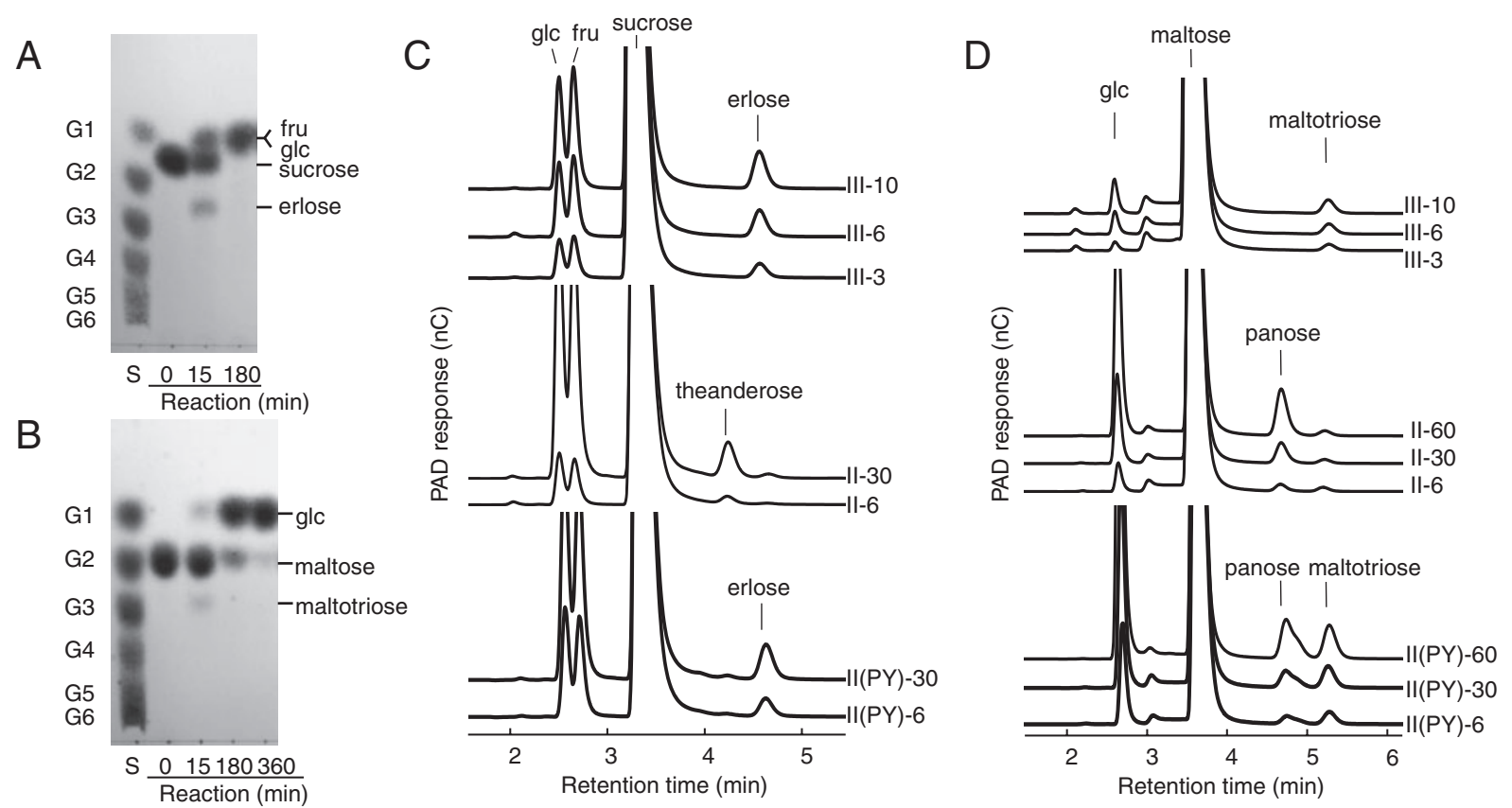

Fig. 3. Analyses of Transglucosylation Products.

A and B, TLC analysis of products on $150 \mathrm{~mm}$ sucrose (A) and maltose (B) during reactions by wild-type HBG-III. S, standards; G1-G6, glucose and maltooligosaccharides to maltohexaose. Products are indicated at the right. C and D, HPAEC-PAD analysis of transglucosylation products on $150 \mathrm{~mm}$ sucrose (C) and maltose (D). The enzymes used and reaction times are indicated at the right side of each chromatogram. III, wild-type HBG-III; II, wild-type HBG-II; II (PY), HBG-II mutant, N226P-H227Y.

$K_{\mathrm{m}}$ for all three substrates than wild-type HBG-III. Particularly, the $k_{\text {cat }}$ for sucrose and the $K_{\mathrm{m}}$ for maltose were 2.5- and 3.5-fold lower respectively than those for wild-type HBG-III.

The kinetic parameters of the wild-type enzymes were drastically changed by mutation (Table 1). Among the HBG-III mutants, only L225V showed higher $k_{\text {cat }}$ for all three substrates than the wild type, but the mutant showed slight reductions in $k_{\text {cat }} / K_{\mathrm{m}}$ values due to the increase in $K_{\mathrm{m}}$. Most of the other HBG-III mutants, particularly those harboring the $\mathrm{P} 226 \rightarrow \mathrm{N}$ mutation, showed lower $k_{\text {cat }}$ values for sucrose. However, they also showed lower $K_{\mathrm{m}}$ values for sucrose, and the $k_{\text {cat }} / K_{\mathrm{m}}$ values of HBG-III mutants were in a range of $35 \%(\mathrm{P} 226 \mathrm{~N})$ and $163 \%(\mathrm{C} 229 \mathrm{~F})$ as compared with the wild type. P226N-H227Y exceptionally showed very low $k_{\text {cat }}$ and $k_{\text {cat }} / K_{\mathrm{m}}$ values. For maltose and maltotriose, $\mathrm{P} 226 \mathrm{~N}$ and its derivatives showed decreases in $k_{\mathrm{cat}} / K_{\mathrm{m}}$, mainly due to a reduction in $k_{\text {cat }}$. The mutants containing the $\mathrm{Y} 227 \rightarrow \mathrm{H}$ mutation displayed elevated $k_{\text {cat }} / K_{\mathrm{m}}, 11$ to 14-fold for maltose and 5-fold for maltotriose, due to the increase in $k_{\text {cat }}$ and the drastic reduction in $K_{\mathrm{m}}$, except for the double mutant, $\mathrm{P} 226 \mathrm{~N}-\mathrm{Y} 227 \mathrm{H}$. The reduced $K_{\mathrm{m}}$ values of $\mathrm{Y} 227 \mathrm{H}$ for the three substrates were even lower than those of wild-type HBG-II. Moreover, the $k_{\text {cat }}$ of the mutant for sucrose was reduced from the wildtype HBG-III, but still higher than that of HBG-II. Hence, $\mathrm{Y} 227 \mathrm{H}$ and its derivatives catalyzed the three substrates with higher $k_{\text {cat }} / K_{\mathrm{m}}$ than wild-type HBG-III and HBG-II.

On the other hand, all the HBG-II mutants showed increased $k_{\text {cat }}$, by 1.6 -fold (N226P for maltotriose) to 3.9-fold ( $\mathrm{H} 227 \mathrm{Y}$ for maltose). However, the $K_{\mathrm{m}}$ values of $\mathrm{H} 227 \mathrm{Y}$ and the double mutant increased, as observed for wild-type HBG-III, which resulted in decreases in $k_{\text {cat }} / K_{\mathrm{m}}$. For instance, the $k_{\text {cat }} / K_{\mathrm{m}}$ values of the double mutant for maltose and maltotriose were very similar to those of wild-type HBG-III. No increase in $K_{\mathrm{m}}$ was observed for the single mutant N226P. Hence, it showed $k_{\text {cat }} / K_{\mathrm{m}}$ elevated by 5.1 -fold for sucrose and 4.2 -fold for maltose.

\section{Reaction at a high substrate concentration}

The reactions products on $150 \mathrm{~mm}$ sucrose and maltose were analyzed (Fig. 3). The TLC results clearly indicated that wild-type HBG-III formed transglucosylation products migrating as erlose and maltotriose at a relatively early stage of the reaction on sucrose and maltose respectively. Both products were degraded by further incubation (Fig. 3A and B). The products were analyzed also with HPAEC-PAD (Fig. 3C and D). HBG-III produced exclusively $\alpha$-1,4-transglucosylation products from sucrose and maltose. On the other hand, HBG-II formed predominantly $\alpha$-1,6-transglucosylation products, theanderose $(\alpha$-Glc- $(1 \rightarrow 6)-\alpha-$ Glc- $(1 \leftrightarrow 2)-\beta$ Fru) and panose, from the substrates respectively, judging by a comparison with authentic substances ${ }^{23}$ ) in the TLC analyses (data not shown) and the HPAECPAD analyses (Fig. 3C and D). Their distinctive regioselectivity in transglucosylation was changed by introducing mutations in the amino acids at 226 and 227. The HBG-II mutant, N226P-H227Y, produced erlose and maltotriose from the substrates (Fig. $3 \mathrm{C}$ and D). In particular, the formation of erlose was much higher than that of theanderose. The accumulation of the transglucosylation products over a long reaction period is summarized in Fig. 4. HBG-III and HBG-II produced $\alpha$ 1,4- and $\alpha$-1,6-transglucosylation products respectively, to approximately 5-6 mM under the reaction conditions, but the HBG-III mutants, P226N and P226N-Y227H, catalyzed not only $\alpha$-1,4-transglucosylation but also $\alpha-1,6$-transglucosylation to produce panose and thean- 

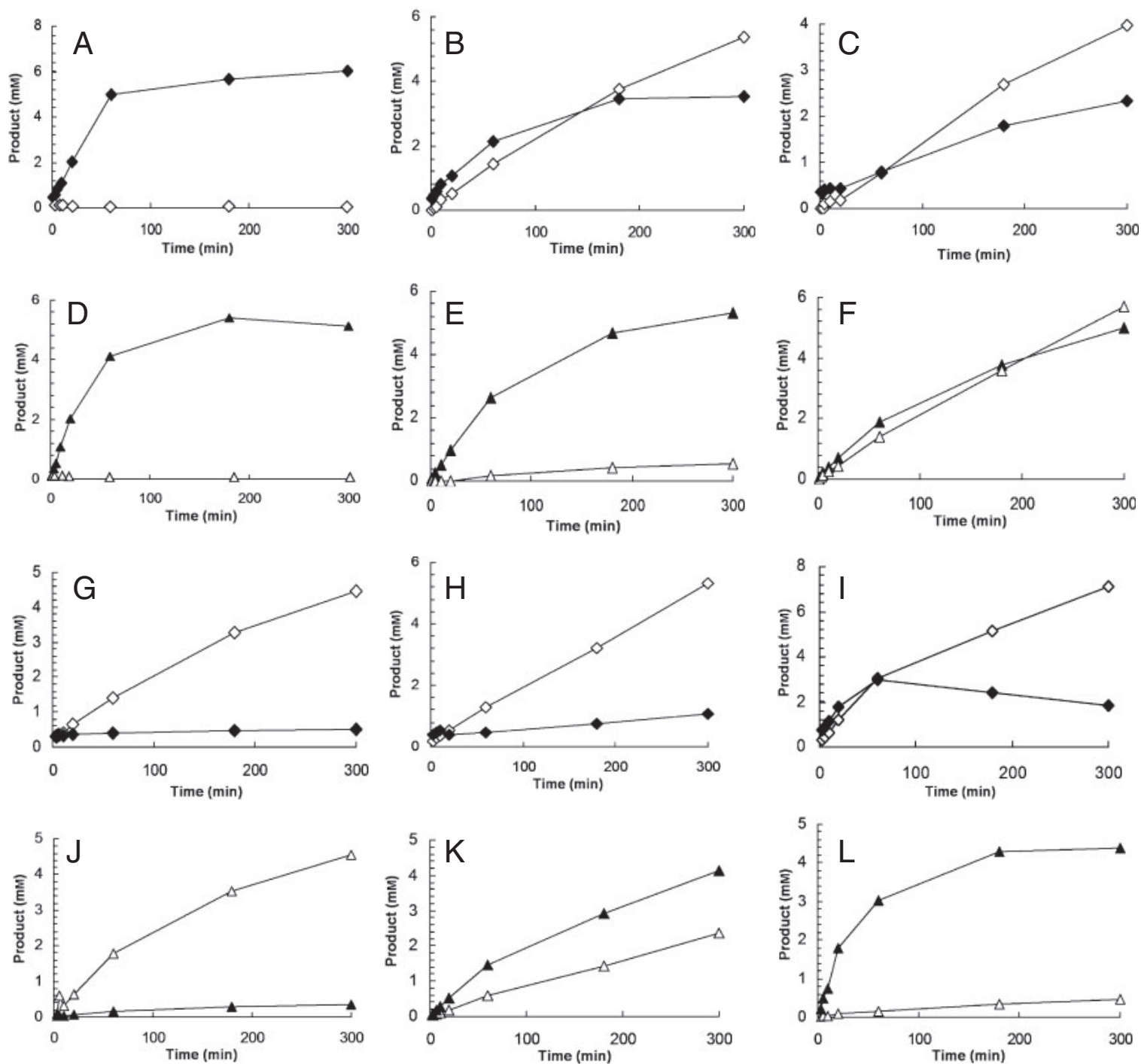

Fig. 4. Progress Curves for Transglucosylation Products.

Products of the reaction on $150 \mathrm{~mm}$ maltose (A, B, C, G, H, and I) and sucrose (D, E, F, J, K, and L) were quantified by HPAEC-PAD analysis. HBG-III derivatives (A and D, wild type; B and E, P226N; C and F, P226N-Y227H) and HBG-II derivatives (G and J, wild type; H and K, N226P; I and L, N226P-H227Y) were used as enzymes. $\diamond$ maltotriose; $\diamond$ panose; $\boldsymbol{\Delta}$ erlose; $\triangle$ theanderose.

derose. On the other hand, accumulation of $\alpha-1,4-$ transglucosylation was observed in the HBG-II mutants, $\mathrm{N} 226 \mathrm{P}$ and N226P-H227Y. In particular, the double mutant accumulated erlose much more than theanderose with sucrose as substrate (Fig. 4L).

By measuring those products with HPAEC-PAD, the initial reaction rates of hydrolysis $\left(v_{\mathrm{h}}\right)$, transglucosylation $\left(v_{\mathrm{tg}}\right)$, and aglycone release $\left(v_{\mathrm{ag}}=v_{\mathrm{h}}+v_{\mathrm{tg}}\right)$ at very early stages of the reaction, when substrate consumption was $0.35 \%$ or less, were determined (Table 2). HBG-III produced exclusively $\alpha$-1,4-transglucosylation products from both substrates, and HBG-II mainly catalyzed $\alpha$ 1,6-transglucosylation, and produced theanderose and panose from sucrose and maltose respectively, even judging from the initial velocities. Wild-type HBG-III showed much higher $v_{\mathrm{ag}}$ than HBG-II. The transglucosylation ratios $\left(r_{\mathrm{tg}}\right)$ of HBG-III were $43 \%$ and $67 \%$ for sucrose and maltose respectively, higher than those of HBG-II (22\% and 59\%).

A variety of reaction velocities and transglucosylation ratios were observed in the reactions of the HBG-III mutants on $150 \mathrm{~mm}$ substrates (Table 2). L225V showed higher $v_{\text {ag }}$ than the wild type for both substrates. The $v_{\text {ag }}$ values of $\mathrm{Y} 227 \mathrm{H}$ and its combination with $\mathrm{I} 228 \mathrm{~L}$ on $150 \mathrm{~mm}$ maltose were also higher than wild-type HBGIII. The mutants containing the $\mathrm{Y} 227 \rightarrow \mathrm{H}$ mutation exhibited very high $r_{\mathrm{tg}}$ values on maltose. The values of $r_{\mathrm{tg}}$ on sucrose of the mutants were lower, but reached $66-74 \%$, significantly higher than that of the wild type (43\%). P226N and its derivatives catalyzed with low $v_{\mathrm{ag}}$. Mutants P226N and P226N-Y227H, however, catalyzed not only $\alpha$-1,4-transglucosylation, but also $\alpha$-1,6-transglucosylation. In particular, P226N-Y227H catalyzed $\alpha$ 1,6-transglucosylation from both substrates. In addition, the rate of $\alpha$-1,6-transglucosylation on maltose of the mutant was even higher than that of $\alpha$-1,4-transglucosylation (Table 2).

Enhancement of the reaction velocities of all three HBG-II mutants was observed not only in the release of glucose, but also in aglycone release (Table 2). The values of $v_{\mathrm{ag}}$ increased by 9.3 -fold at maximum. All three mutants catalyzed $\alpha$-1,4-transglucosylation from sucrose as substrate, while wild-type HBG-II catalyzed predominantly $\alpha$-1,6-transglucosylation. The N226 $\rightarrow \mathrm{P}$ mutation particularly altered regioselectivity, even on maltose. Single mutant N226P increased $r_{\text {tg }}$ (41\% on 
Table 2. Reaction Velocities on $150 \mathrm{~mm}$ Substrates and Transglucosylation Ratios

\begin{tabular}{|c|c|c|c|c|c|c|c|c|}
\hline \multirow{3}{*}{ Enzyme } & \multicolumn{4}{|c|}{ Sucrose } & \multicolumn{4}{|c|}{ Maltose } \\
\hline & $v_{\mathrm{ag}}$ & $v_{4 \operatorname{tg}}{ }^{\mathrm{a}}$ & $v_{6 \mathrm{tg}} \mathrm{b}$ & \multirow{2}{*}{$\begin{array}{l}r_{\mathrm{tg}} \\
(\%)\end{array}$} & $v_{\mathrm{ag}}$ & $v_{4 \operatorname{tg}}{ }^{\mathrm{a}}$ & $v_{6 \mathrm{tg}}{ }^{\mathrm{b}}$ & \multirow{2}{*}{$\begin{array}{l}r_{\mathrm{tg}} \\
(\%)\end{array}$} \\
\hline & \multicolumn{3}{|c|}{$\left(\mu \mathrm{mol} \mathrm{min} \mathrm{mg}^{-1} \mathrm{mg}^{-1}\right)$} & & \multicolumn{3}{|c|}{$\left(\mu \mathrm{mol} \mathrm{min}{ }^{-1} \mathrm{mg}^{-1}\right)$} & \\
\hline \multicolumn{9}{|l|}{ HBG-III } \\
\hline Wild type & 198 & 85.5 & nd & 43 & 45.0 & 30.4 & nd & 68 \\
\hline L225V & 256 & 86.2 & nd & 34 & 126 & 86.0 & nd & 69 \\
\hline L225I & 196 & 82.1 & nd & 42 & 81.0 & 58.1 & nd & 72 \\
\hline $\mathrm{P} 226 \mathrm{~N}$ & 19.3 & 5.7 & nd & 29 & 4.7 & 1.8 & 1.2 & 63 \\
\hline $\mathrm{Y} 227 \mathrm{H}$ & 97.2 & 67.0 & nd & 69 & 63.4 & 63.2 & nd & 100 \\
\hline $\mathrm{I} 228 \mathrm{~L}$ & 114 & 37.7 & nd & 33 & 22.6 & 7.3 & nd & 32 \\
\hline $\mathrm{I} 228 \mathrm{M}$ & 87.3 & 5.7 & nd & 7 & 67.5 & 29.0 & nd & 43 \\
\hline $\mathrm{C} 229 \mathrm{~F}$ & 213 & 45.5 & nd & 21 & 59.7 & 26.9 & nd & 45 \\
\hline L225I-P226N & 83.5 & 28.0 & nd & 33 & 23.2 & 21.5 & nd & 93 \\
\hline P226N-Y227H & 2.8 & 1.3 & 0.8 & 74 & 0.8 & 0.3 & 0.5 & 97 \\
\hline Y227H-I228L & 72.1 & 47.8 & nd & 66 & 95.0 & 95.0 & nd & 100 \\
\hline Y227H-I228M & 81.7 & 59.5 & nd & 73 & 34.3 & 26.0 & nd & 76 \\
\hline \multicolumn{9}{|l|}{ HBG-II } \\
\hline Wild type & 41.2 & nd & 8.9 & 22 & 8.2 & nd & 4.9 & 59 \\
\hline $\mathrm{N} 226 \mathrm{P}$ & 105 & 29.4 & 17.6 & 41 & 35.9 & 4.3 & 21.4 & 72 \\
\hline $\mathrm{H} 227 \mathrm{Y}$ & 152 & 6.7 & 10.0 & 9 & 76.8 & nd & 28.6 & 37 \\
\hline N226P-H227Y & 143 & 31.0 & 1.7 & 30 & 59.2 & 9.3 & 9.1 & 31 \\
\hline
\end{tabular}

${ }^{a}$ Velocity of $\alpha$-1,4-transglucosylation yielding erlose and maltotriose from sucrose and maltose respectively.

${ }^{b}$ Velocity of $\alpha-1,6$-transglucosylation yielding theanderose and panose from the substrates respectively.

nd, Not determined due to velocities too low.

sucrose, $72 \%$ on maltose), while the two others containing $\mathrm{H} 227 \rightarrow \mathrm{Y}$ mutation decreased in these ratios significantly.

\section{Discussion}

$\alpha$-Glucosidase isozymes from the European honeybee exhibit many differences in substrate specificity, transglucosylation, and other kinetic behaviors. HBG-II, purified from honeybees, has broad substrate specificity with activity lower than HBG-I and HBG-III. ${ }^{9)}$ In contrast, HBG-III acts specifically on sucrose, maltose, and maltotriose with high $k_{\text {cat }}$ and $K_{\mathrm{m}} \cdot{ }^{5)}$ Recombinant wild-type HBG-II and HBG-III, investigated in this study, exhibited characteristics similar to those of the native enzymes. The kinetic parameters, determined at low substrate concentrations, were in good agreement with those previously reported. ${ }^{12}$ ) In this study, rate determination at a high substrate concentration, $150 \mathrm{mM}$, revealed the high transglucosylation ability of HBG-III and the regioselectivity of the enzymes in transglucosylation.

The distinctive differences between HBG-II and HBG-III in substrate specificity, kinetic parameters, and transglucosylation were drastically changed by introducing single and double mutations in conserved region II, particularly at Pro226-Tyr227 of HBG-III and the equivalent. By replacement of the corresponding Asn226-His227 of HBG-II by Pro-Tyr, the lower $k_{\text {cat }}$ and $K_{\mathrm{m}}$ of HBG-II were elevated to the level of HBG-III or even higher. The $k_{\mathrm{cat}} / K_{\mathrm{m}}$ values of the resulting double mutant on maltose and maltotriose were in a good agreement with those of HBG-III (Table 1). The regioselectivities of HBG-III and HBG-II were altered toward the other by exchanging residues (Fig. 4 and Table 2). Therefore, it can be concluded that Pro226Tyr227 of HBG-III and the equivalents of HBG-II are a determinant distinguishing the two enzymes.
The crucial amino acids were also effective as HBG-I determinants. Two mutants, Y227H of HBG-III and N226P of HBG-II, which shared Pro-His at the positions with HBG- $\mathrm{I}^{12)}$ and JBG-I ${ }^{10)}$ indicated in Fig. 1, exhibited enzymatic properties similar to HBG-I. ${ }^{7,8)}$ Both mutants showed high activity particularly on maltooligosaccharides (Table 1), and the substrate specificity toward maltose was very high (Fig. 2). The $\alpha-1,6-$ regioselectivity of HBG-II was changed to $\alpha-1,4$ by the $\mathrm{N} 226 \rightarrow \mathrm{P}$ mutation (Fig. 4 and Table 2). In addition, drastic increases in $r_{\mathrm{tg}}$ were observed in both mutant enzymes. All of the features the mutants acquired are similar to those of HBG-I. ${ }^{7,8)}$

The roles of the amino acids in enzymatic function can be concluded to be as follows: His 227 and Tyr227 are involved in substrate preferences for maltose and sucrose respectively. The residues at position 226 are a determinant of regioselectivity in transglucosylation. Pro226 and Asn226 respectively are associated with $\alpha$ 1,4- and $\alpha$-1,6-transglucosylation. A high transglucosylation ratio can be achieved in mutants harboring Pro226-His227, which resemble HBG-I in high transglucosylation ratios and amino acids at the corresponding positions. ${ }^{8,12)}$

The residues corresponding to Pro226-Tyr227 in HBG-III are in conserved sequence region II of the GH-13 enzymes. ${ }^{11-14)}$ Conserved regions I-IV include seven invariant amino acids essential to activity. There are variations in the other amino acids in these regions, and some of them are responsible for the specific properties of enzymes. ${ }^{14)}$ The residue C-terminally adjacent to the catalytic Asp in region II, corresponding to Ala224 of HBG-III and HBG-II, is a determinant of the substrate specificity of GH-13 enzymes. ${ }^{16,18)}$ The two following amino acid residues, equivalent to Pro226-Tyr227 of HBG-III, have been pointed out as the characteristic signature of GH-13 enzymes. ${ }^{14)}$ According to a recent subfamily classification of GH-13 
enzymes, ${ }^{24)}$ the sequence motif DAxxH (corresponding Asp223-Tyr227 of HBG-III as shown in Fig. 1) is often found in subfamilies $1,2,5,15,17,19,24,27,28,31$, 32 , and 36, which include $\alpha$-1,4-linkage-specific enzymes such as $\alpha$-amylases, cyclomaltodextrin glucanotransferases, and $\alpha$-glucosidases. Most $\alpha$-amylases and cyclomaltodextrin glucanotransferases have dipeptide Lys-His at the C-terminal end of the motif. The corresponding His 210 of Taka-amylase makes a hydrogen bond with $2 \mathrm{OH}$ of the 6-deoxyglucose moiety of an acarbose derivative at subsite +1 in a complex structure. $^{25)}$ The equivalent His180 of Bacillus subtilis $\alpha$ amylase is also involved in the hydrogen bond at the same position. ${ }^{26)}$ Our biochemical data clearly indicate the involvement of the corresponding His227 of HBG-II in the binding of maltooligosaccharides. The preference of HBG-II for maltooligosaccharides is most likely due to the hydrogen-bond formation of His227 with a glucose residue of maltooligosaccharides at subsite +1 .

We found that the Tyr residue situated at position 227 in HBG-III is involved in sucrose preference with drastic increases in both $k_{\text {cat }}$ and $K_{\mathrm{m}}$. The sequence motif DAxxY/F (corresponding to Asp223-Tyr227 of HBGIII) frequently occurs in GH-13 enzymes belonging to subfamilies 4 (sucrose hydrolases), 16 (trehalose synthases), 17 ( $\alpha$-glucosidases), 18 (sucrose phosphorylases), and 23 ( $\alpha$-glucosidases). The protein structures of Tyr-harboring enzymes, sucrose hydrolase derived from Xanthomonas campestris ${ }^{27)}$ and sucrose phosphorylase from Bifidobacterium adolescentis, ${ }^{28)}$ indicate that the side chain of Tyr has no direct contact with a fructose moiety of sucrose. It is accommodated on the far side of the pocket from the equivalent residues, ${ }^{27)}$ as observed for the isomaltotriose-binding mode of dextran glucosidase. ${ }^{29)}$ In contrast, maltooligosaccharides are expected to bind closely to the residue. ${ }^{27,30)}$ Introduction of the bulky Tyr residue at this position probably causes steric hindrance in the binding of substrates, and substratebinding in a distorted manner might increase the energy state of the Michaelis complex, resulting in increases in $K_{\mathrm{m}}$ and $k_{\text {cat }}$

\section{Acknowledgment}

We thank Mr. Tomohiro Hirose of Instrumental Analysis Division, the Equipment Management Center, Creative Research Institution, Hokkaido University for amino acid analysis.

\section{References}

1) Chiba S, "Handbook of Amylases and Related Enzymes," ed. The Amylase Research Society of Japan, Pergamon Press, Oxford, pp. 104-116 (1998)

2) Chiba S, Biosci. Biotechnol. Biochem., 61, 1233-1239 (1997).

3) Henrissat B and Davies GJ, Curr. Opin. Struct. Biol., 7, 637644 (1997)
4) Takewaki S, Chiba S, Kimura A, Matsui H, and Koike Y, Agric. Biol. Chem., 44, 731-740 (1980).

5) Nishimoto M, Kubota M, Tsuji M, Mori H, Kimura A, Matsui H, and Chiba S, Biosci. Biotechnol. Biochem., 65, 1610-1616 (2001).

6) Kubota M, Tsuji M, Nishimoto M, Wongchawalit J, Okuyama M, Mori H, Matsui H, Surarit R, Svasti J, Kimura A, and Chiba S, Biosci. Biotechnol. Biochem., 68, 2346-2352 (2004).

7) Kimura A, Takewaki S, Matsui H, Kubota M, and Chiba S, J. Biochem., 107, 762-768 (1990).

8) Kimura A, Yoshida-Kitahara F, and Chiba S, Agric. Biol. Chem., 51, 1859-1864 (1987).

9) Takewaki S, Kimura A, Kubota M, and Chiba S, Biosci. Biotechnol. Biochem., 57, 1508-1513 (1993).

10) Wongchawalit J, Yamamoto $T$, Nakai H, Kim YM, Sato N, Nishimoto M, Okuyama M, Mori H, Saji O, Chanchao C, Wongsiri S, Surarit R, Svasti J, Chiba S, and Kimura A, Biosci. Biotechnol. Biochem., 70, 2889-2898 (2006).

11) Ohashi K, Sawata M, Takeuchi H, Natori S, and Kubo T, Biochem. Biophys. Res. Commun., 221, 380-385 (1996).

12) Nishimoto $M$, Mori $H$, Moteki $T$, Takamura $Y$, Iwai $G$, Miyaguchi Y, Okuyama M, Wongchawalit J, Surarit R, Svasti J, Kimura A, and Chiba S, Biosci. Biotechnol. Biochem., 71, 1703-1716 (2007).

13) Svensson B, Plant Mol. Biol., 25, 141-157 (1994).

14) MacGregor EA, Janeček Š , and Svensson B, Biochim. Biophys. Acta, 1546, 1-20 (2001).

15) Inohara-Ochiai M, Nakayama $T$, Goto R, Nakao M, Ueda T, and Shibano Y, J. Biol. Chem., 272, 1601-1607 (1997).

16) Yamamoto K, Nakayama A, Yamamoto Y, and Tabata S, Eur. J. Biochem., 271, 3414-3420 (2004).

17) Saburi W, Mori H, Saito S, Okuyama M, and Kimura A, Biochim. Biophys. Acta, 1764, 688-698 (2006).

18) Tsujimoto Y, Tanaka H, Takemura R, Yokogawa T, Shimonaka A, Matsui H, Kashiwabara S, Watanabe K, and Suzuki Y, J. Biochem., 142, 87-93 (2007).

19) Lin-Cereghino J, Wong WW, Xiong S, Giang W, Luong LT, Vu J, Johnson SD, and Lin-Cereghino GP, BioTechniques, 38, 4448 (2005).

20) Laemmli UK, Nature, 227, 680-685 (1970).

21) Sugiura M and Hirano K, Clin. Chim. Acta, 75, 387-391 (1977).

22) Kobayashi M, Hondoh H, Mori H, Saburi W, Okuyama M, and Kimura A, Biosci. Biotechnol. Biochem., 75, 1557-1563 (2011).

23) Chiba S, Asada-Komatsu Y, Kimura A, and Kawashima K, Agric. Biol. Chem., 48, 1173-1178 (1984).

24) Stam MR, Danchin EG, Rancurel C, Coutinho PM, and Henrissat B, Protein Eng. Des. Sel., 19, 555-562 (2006).

25) Brzozowski AM and Davies GJ, Biochemistry, 36, 1083710845 (1997).

26) Fujimoto $Z$, Takase $K$, Doui N, Momma M, Matsumoto T, and Mizuno H, J. Mol. Biol., 277, 393-407 (1998).

27) Kim MI, Kim HS, Jung J, and Rhee S, J. Mol. Biol., 380, 636647 (2008).

28) Mirza O, Skov LK, Sprogoe D, van den Broek LA, Beldman G, Kastrup JS, and Gajhede M, J. Biol. Chem., 281, 35576-35584 (2006).

29) Hondoh H, Saburi W, Mori H, Okuyama M, Nakada T, Matsuura Y, and Kimura A, J. Mol. Biol., 378, 913-922 (2008).

30) Skov LK, Mirza O, Sprogoe D, Dar I, Remaud-Simeon M, Albenne C, Monsan P, and Gajhede M, J. Biol. Chem., 227, 47741-47747 (2002).

31) Ravaud S, Robert X, Watzlawick H, Haser R, Mattes R, and Aghajari N, J. Biol. Chem., 282, 28126-28136 (2007). 